\title{
The Role of Real-Time Tissue Elastography and Gray-Scale Ultrasound Histogram Analysis in the Diagnosis of Patients With Sjögren's Syndrome
}

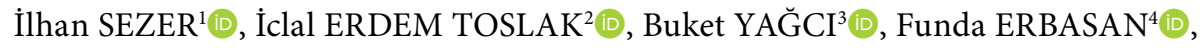 \\ Ayşe AYAN $^{5}\left(\right.$, Uğur KARASU ${ }^{6}$ \\ ${ }^{1}$ Department of Physical Medicine and Rehabilitation, Division of Rheumatology, Akdeniz University Faculty of Medicine, Antalya, Turkey \\ ${ }^{2}$ Department of Radiology, Antalya Training and Research Hospital, Antalya, Turkey \\ ${ }^{3}$ Department of Radiology, Kastamonu State Hospital, Kastamonu, Turkey \\ ${ }^{4}$ Department of Internal Medicine, Division of Rheumatology, Akdeniz University Faculty of Medicine, Antalya, Turkey \\ ${ }^{5}$ Department of Rheumatology, Antalya Training and Research Hospital, Antalya, Turkey \\ ${ }^{6}$ Department of Internal Medicine, Division of Rheumatology, Pamukkale University Faculty of Medicine, Denizli, Turkey
}

\begin{abstract}
Objectives: This study aims to evaluate gray-scale histogram analysis of B-mode ultrasound (US) images and US elastographic features of the parotid glands in patients with Sjögren's syndrome (SS) and to explore relationships with the ultrasonographic and disease activity scores in the light of histopathological findings.

Patients and methods: A total of 57 consecutive female patients (mean age $47.9 \pm 10.4$ years; range 25 to 76 years) with a diagnosis of SS and 48 healthy female individuals (mean age $51.1 \pm 10.8$ years; range 20 to 70 years) underwent parotid ultrasonography and real-time tissue elastography imaging. Quantitative measurements of gray-scale US images were performed using the histogram software of the scanner. The histogram ratios of the parotid glands from both sides were obtained (histogram ratio; mean gray-scale histogram parotid/mean gray-scale histogram fat). Strain ratio (SR; ratio of fat to gland parenchyma) was calculated from the color-coded images. Subjective B-mode US scoring of electronically recorded grayscale US images was performed by two radiologists independently for intra- and inter-observer agreement. Subjective assessments, quantitative measurements, and clinical parameters were compared.

Results: The SR of the patient group (1.4 \pm 0.8 right side, $1.5 \pm 0.9$ left side) was significantly higher than that of the control group (1.0 \pm 0.3 right side, $1.1 \pm 0.3$ left side) $(p<0.05)$. The gray-scale histogram ratio of the patient group $(1.3 \pm 0.5$ right side, $1.4 \pm 0.9$ left side) was lower than that of the control group ( $1.8 \pm 0.7$ right side, $1.9 \pm 0.7$ left side) ( $p<0.05$ ). Receiver-operating-characteristics curve yielded $66 \%$ sensitivity for both sides and $50 \%$ and $52 \%$ specificity for the right and left sides, respectively, for a cut-off SR of $1.02 ; 76 \%$ and $86 \%$ sensitivity for the right and left sides, respectively, and $63 \%$ specificity for both sides for a cut-off histogram ratio of 1.35 . The quantitative histogram ratio method had a higher positivity rate for the diagnosis of abnormal parotid glands than subjective assessments of US images.

Conclusion: Sonoelastography and gray-scale histogram analysis of the parotid glands may be used as auxiliary tools to detect parotid gland sonographic abnormalities in patients with SS.

Keywords: Elastography; Sjögren's syndrome; ultrasonography.
\end{abstract}

Sjögren's syndrome (SS) is a chronic systemic inflammatory disease characterized by lymphocytic infiltration of all exocrine glands including the salivary and lacrimal glands. The leading clinical symptoms are xerostomia and xerophthalmia, although half of the

Received: July 23, 2018 Accepted: December 25, 2018 Published online: March 28, 2019

Correspondence: Funda Erbasan, MD. Akdeniz Üniversitesi Tıp Fakültesi İç Hastalıkları Anabilim Dalı, Romatoloji Bilim Dalı, 7070 Konyaaltı, Antalya, Turkey. Tel: +90 532 - 3019661 e-mail: funda@yahoo.com

Citation:

Sezer I, Erdem Toslak I, Yağcı B, Erbasan F, Ayan A, Karasu U. The role of real-time tissue elastography and gray-scale ultrasound histogram analysis in the diagnosis of patients with Sjögren's syndrome. Arch Rheumatol 2019;34(4):371-379. 
patients also have associated extra-glandular systemic symptoms including arthritis, lung, central nervous system, and renal involvement. ${ }^{1}$ The prevalence of the disease varies from 0.2 to $2.7 \% .^{2}$ This variability of prevalence could be a result of the diversity of the classification criteria or insufficiency of the diagnostic tools. Diagnosis is usually based on the AmericanEuropean Consensus Group (AECG) criteria for the classification of SS, which were proposed in 2002 , and the classification criteria were set by the American College of Rheumatology (ACR) in 2012.3.4 Diagnostic criteria include clinical and laboratory data as well as direct and indirect examination methods for the salivary glands. Salivary gland assessment methods include the measurement of the amount of saliva, using imaging techniques such as sialography and scintigraphy, and minor salivary gland biopsy. These tools have the disadvantages of variability in sensitivity and specificity rates, exposure to radioactive materials or complications of invasive procedures. ${ }^{5}$ Past studies using ultrasound (US) imaging for the diagnosis of SS have shown promising results. ${ }^{6}$ However, US evaluation in most of those studies was visual and therefore, subjective. Gray-scale histogram analysis of US images is a more objective method providing quantitative information about the echogenicity state of the glands based on echo-intensity levels within a range of 0 to 255 gray shades, thus eliminating subjective, operator-dependent features of the commonly used visual US evaluation method.? There are also studies in the literature using US elastography of salivary glands for the diagnosis of SS based on the tissue stiffness, which is a prominent feature of the diseased glands due to the fibrotic changes associated with the disease. ${ }^{5}$ To the best of our knowledge, there is no study in the literature that has quantified the echogenicity of the glands with gray-scale histogram analysis and the stiffness of glands using sonoelastography and then correlated these results with histopathological findings in patients with SS. Therefore, in this study, we aimed to evaluate gray-scale histogram analysis of B-mode US images and US elastographic features of the parotid glands in patients with SS and to explore relationships with the ultrasonographic and disease activity scores in the light of histopathological findings.

\section{PATIENTS AND METHODS}

Between January 2016 and December 2016, a total of 57 consecutive female patients (mean age $47.9 \pm 10.4$ years; range 25 to 76 years) who were referred to rheumatology outpatient clinics of Antalya Training and Research Hospital with complaints of dry mouth and dry eye and diagnosed as SS based on ACR 2012 classification criteria were prospectively included in this study. ${ }^{4}$ A control group was formed of 48 healthy female individuals (mean age $51.1 \pm 10.8$ years; range 20 to 70 years). A complete physical examination was applied to all participants, including acute phase reactants (erythrocyte sedimentation rate, C-reactive protein), and complete blood count. Immunological tests included antinuclear antibodies (ANA) determined by indirect immunofluorescence assay on Hep-2 cells (a titer of 160 was considered positive), rheumatoid factor (RF) detected by nephelometry, and antibodies to the extractable nuclear antigens (Ro/SS type A [SS-A] and La/SS type B [SS-B], detected by enzyme-linked immunosorbent assay). Exclusion criteria were the use of medications with the potential to influence saliva production, a past medical history of radiation exposure to the head and neck region, hepatitis $B$, hepatitis $C$, human immunodeficiency virus infections, sarcoidosis, lymphoma, and graft versus host reaction. Lacrimation was evaluated using the Schirmer ( $<5 \mathrm{~mm}$ in 5 minutes) and break-up time methods. For the evaluation of disease activity, European League Against Rheumatism SS disease activity index $(E S S D A I)^{8}$ and patient reported index $(\text { ESSPRI })^{9}$ were used. The ESSPRI was calculated using a 0-10 numeric scale divided by three based on visual estimation, and the SS disease activity index was decided based on systemic involvement (0-123); slow-activity, (ESSDAI <5), moderate-activity $(5 \leq$ ESSDAI $\leq 13)$, high-activity (ESSDAI 214 ). Minor salivary gland biopsy samples were taken from the lower lips via a minor incision. Histopathological analyses were assessed using the Chisholm classification. ${ }^{10}$ The control group individuals were selected from a healthy population and had no dryness or systemic symptoms. The study protocol was approved by the Antalya Training and Research Hospital Ethics Committee. A written informed consent was obtained from each participant. 
The study was conducted in accordance with the principles of the Declaration of Helsinki.

All participants underwent an US examination in the Radiology Department using the same scanner (Hi-Vision Preirus; Hitachi Medical Corp., Tokyo, Japan) equipped with real-time tissue elastography imaging software and a gray-scale histogram function. All US examinations were performed by the same radiologist with four years of experience on US imaging and three years of experience on elastography imaging, and who was blinded to the diagnoses. All examinations were performed using a high frequency $(8-15 \mathrm{MHz})$ linear array transducer with the participants in the supine position. Initial diagnostic focused longitudinal and transverse US images of the parotid gland were recorded electronically in the form of dynamic and static images. The size, shape, margin, echogenicity of the glands and potential mass lesions, if any, were included while recording the images. Echogenicity of the glands was decided relative to the thyroid gland echogenicity based on previous descriptions in the literature. ${ }^{11}$

To evaluate the echogenicity of the glands quantitatively, gray-scale histogram measurements were performed within a range of distribution of pixel gray-levels from 0 to 255 (0: black, 255: white) using B-mode US images similar to the technique used by Maeda et al. ${ }^{12}$ To prevent any disparity in gray-scale echogenicity of the parotid glands, reference measurements from the adjacent subcutaneous fat tissue were performed. All measurements were taken from the longitudinal still images using dedicated software present in the scanner. The method used to take the measurements was as follows: First, a circular region of interest (ROI) was defined on the most representative parts of the parotid glandular parenchyma. Then, a second ROI was defined on the adjacent subcutaneous fat tissue as the reference tissue. The mean values of pixels, as quantitative histogram parameters, of both ROIs were automatically displayed on the screen of the US scanner. Finally, the histogram ratio of the parotid gland was calculated by dividing the mean gray-scale histogram value of the parotid gland by the mean gray-scale histogram value of subcutaneous fat (mean gray-scale histogram parotid/mean gray-scale histogram fat) for each side of all individuals (Figure 1a, b). All measurements were repeated three times and the average was recorded.

Free hand elastography was performed in the same session as the diagnostic focused US examination. To provide standardization, depth, focus position, and gain settings were adjusted to be the same for all individuals. Elastographic images were acquired using the freehand repeated manual compression technique in which the transducer was continuously compressed and decompressed over the skin of the subject. A gentle axial movement of the transducer was sufficient to obtain optimal waveforms. The elastograms were then obtained, with split-screen mode, one displaying the real-time conventional $\mathrm{B}$-mode image and the other screen displaying
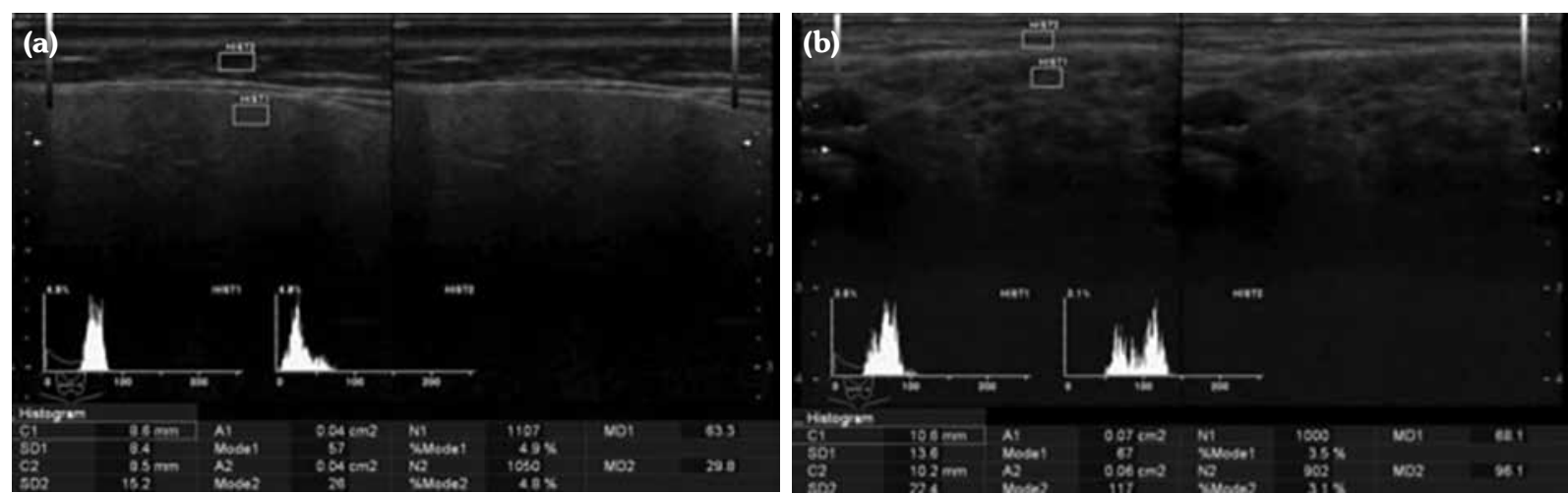

Figure 1. (a) Sample ultrasonographic image for gray-scale histogram analysis of a 46-year-old healthy female individual. First region of interest was placed on parotid gland and second region of interest was placed on subcutaneous tissue. Mean histogram ratio was calculated as 2.12. (b) Mean histogram ratio was calculated as 0.71 in a 69-year-old female patient with Sjögren's disease. B-mode ultrasound score was rated as Grade 4 in a homogenous gland with numerous hypoechogenic areas ( $>6 \mathrm{~mm})$. 
the strain elastography images. The elastograms were displayed through 256 colormapping with a scale ranging from red (the softest component, maximum strain) to green (intermediate component, average strain) to blue (the hardest component, no strain) depending on the tissue displacement. The speed of compression and transducer pressure were accepted as ideal when a mixture of red and green was observed on the subcutaneous fat layer and blue was observed on the neighboring muscle layer. Care was taken not to interrupt color encoding. It took approximately two-three minutes for each case to acquire the elastographic imaging.

The evaluation of elastography images was performed by radiologists at the same time as the images were obtained. Strain ratio (SR) measurements of each gland were taken from the color-coded images. With the assumption that fat has relatively stable stiffness, subcutaneous fat was accepted as the reference area with relatively constant stiffness. A circular ROI was defined on the most representative parts of the parotid glandular parenchyma and a second ROI was defined on the adjacent subcutaneous fatty tissue on the elastograms anteriorly. The SR was then automatically calculated by the software present in the scanner that corresponded to the ratio of fat to gland parenchyma based on previous descriptions in the literature (Figure 2). ${ }^{13}$

All recorded images were transferred to a separate workstation for subjective assessment of B-mode US images in terms of parenchymal echostructure and echogenicity of the glands. The images were reviewed independently by two radiologists, each with more than four years

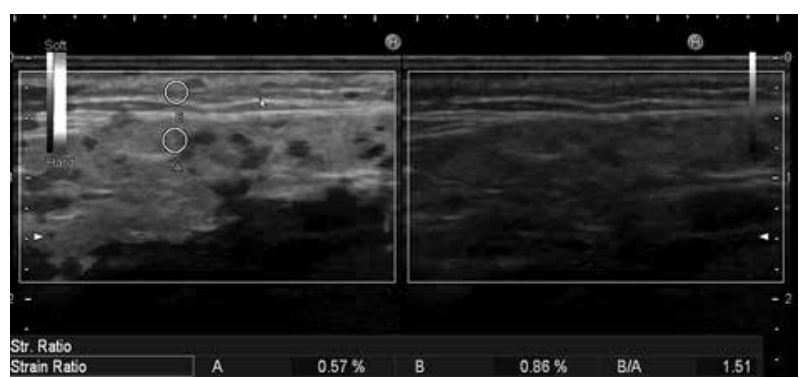

Figure 2. Gray-scale (on right side) and color-coded elastography (on left side) ultrasonographic images of a 49-year-old female patient with Sjögren's disease. Strain ratio was calculated as 1.51 . B-mode ultrasound score was rated as Grade 3 in a homogenous gland with multiple hypoechogenic areas $(2-6 \mathrm{~mm})$ and echogenic bands. of experience, and who were both blinded to all the information of the participants. At two weeks after the initial evaluation, each radiologist reviewed all the images once again. In cases where there was disagreement, the two radiologists convened and reached a consensus in a separate section. The echostructure of the glands was determined based on a rating system previously described by Hofauer et al. ${ }^{5}$ According to this, the echostructure of the parenchyma was subjectively stratified as four grades as follows: Grade 0, homogenous; Grade 1, small hypoechogenic areas without echogenic bands; Grade 2, multiple hypoechogenic areas $(<2 \mathrm{~mm})$ with echogenic bands; Grade 3, multiple hypoechogenic areas (2-6 mm) with echogenic bands; Grade 4, multiple hypoechogenic areas ( $>6 \mathrm{~mm}$ ). Any score $\geq 2$ was accepted as abnormal based on the optimal cut-off values reported in previous studies in the literature. ${ }^{5}$

\section{Statistical analysis}

The statistical analyses were performed using statistical analysis system (SAS) 9.4 software (SAS Institute, Cary, North Carolina, USA). Descriptive statistics were presented as number (n) and percentage (\%), and the differences between groups were assessed for statistical significance using the Student's t-test. Cronbach's alpha was used in the analysis of inter- and intrarater reliability for $\mathrm{B}$-mode US scoring (BMUS) (>0.9=excellent, $>0.8=$ good, $>0.7=$ acceptable, $>0.6$ =questionable, $\quad>0.5=$ weak, $\leq 0.5=$ not acceptable). Receiver operating characteristics (ROC) curves were formed for gray-scale histograms and SRs to differentiate normal from abnormal glands. Sensitivity, specificity, positive predictive value, and negative predictive value were determined for various cut-points. Multidimensional analysis was used to compute the relationships between diagnostic variables. All differences associated with a $p$ value of $<0.05$ were considered statistically significant.

\section{RESULTS}

Evaluation was performed of a total of 57 female patients with a mean age of $47.9 \pm 10.4$ years and 48 healthy female individuals with a mean age of $51.1 \pm 10.8$ years. There was no statistically significant difference between the two groups $(p \geq 0.05)$. 
In the patient group, the mean duration of the complaints was $62.0 \pm 55.2$ months (range, 1-240 months) and the mean time to diagnosis was $31.9 \pm 27.6$ months (range, 1-108 months). The patient characteristics are summarized in Tables 1 and 2 . The diagnostic parameters were evaluated using multidimensional analysis (Figure 3). There was a statistically significant positive correlation between ANA autoantibodies and elastography values $(p=0.008)$. There was no correlation between any of the acute phase reactants, SS-A, SS-B, Ro52, RF levels, ESSPRI, ESSDAI, or minor salivary gland biopsy score parameters with the histogram or elastography values of the parotid glands.

In the gray-scale histogram measurements, the mean gray-scale histogram ratios of the right and left-side parotid glands were $1.3 \pm 0.5$ and $1.4 \pm 0.9$, respectively, in the patient group and $1.8 \pm 0.7$ and $1.9 \pm 0.7$, respectively, in the control group. The mean B-mode US images, gray-scale

Table 1. Distribution of diagnostic parameters for diagnosis of Sjögren's syndrome

\begin{tabular}{lcc}
\hline Parameter & Mean \pm SD & Min-Max \\
\hline Duration of the complaints (months) & $62.0 \pm 55.2$ & $1-240$ \\
Time to diagnosis (months) & $31.9 \pm 27.6$ & $1-108$ \\
Patient report index (0-10) & $5.9 \pm 2.2$ & $0-10$ \\
ESSDAI & $3.8 \pm 2.5$ & $0-8$ \\
Schirmer test (mm) & $5.2 \pm 6.3$ & $0-30$ \\
Break-up time (sec) & $5.3 \pm 6.3$ & $1-20$ \\
ESR (mm/h) & $30.4 \pm 22.1$ & $3-118$ \\
C-reactive protein (mg/L) & $8.0 \pm 15.0$ & $0.5-85$ \\
\hline SD: Standard deviation; Min: Minimum; Max: Maximum; ESSDAI: \\
European League Against Rheumatism Sjögren's syndrome disease activ- \\
ity index; ESR: Erythrocyte sedimentation rate. \\
\hline
\end{tabular}

Table 2. Distribution of diagnostic parameters for diagnosis of Sjögren's syndrome

\begin{tabular}{lcc}
\hline Parameter & $\mathrm{n}$ & $\%$ \\
\hline Focus score $\geq 1 \quad(0-4)$ & 52 & 91 \\
Antinuclear antibodies & 46 & 80 \\
Anti-SS-A & 27 & 43 \\
Anti-SS-B & 16 & 28 \\
Anti-Ro52 & 26 & 45 \\
Rheumatoid factor & 19 & 33 \\
\hline SS-A: Sjögren's syndrome type A; SS-B: Sjögren's syndrome type B.
\end{tabular}

histograms, and sonoelastography measurements are summarized in Table 3. The mean gray-scale histogram ratios of patients were significantly lower than those of the control group $(p<0.001$ for the right side and $p=0.002$ for the left side). ROC analysis yielded area under the curve values of 0.763 and 0.818 for the right and left sides, respectively, demonstrating reasonable and strong discrimination of normal from abnormal glands for the right and left sides, respectively. For the optimal cut-off value of $1.35,76 \%$ sensitivity and $63 \%$ specificity were determined for the right side while sensitivity was found to be $86 \%$ and specificity was found to be $63 \%$ for the left side.

In the sonoelastography measurements, the mean SR of the right and left-side parotid glands was $1.4 \pm 0.8$ and $1.5 \pm 0.9$, respectively, in the patient group and $1.0 \pm 0.3$ and $1.1 \pm 0.3$, respectively, in the control group. The mean SR of the patient group was significantly higher than that of the control group $(p=0.009$ right side, $\mathrm{p}=0.020$ left side). The c-statistic for the SR was 0.628 for the right and 0.616 for the left side, which means poor discrimination of normal from abnormal glands. For the left side, for the optimal cut-off value of 1.05 , sensitivity was $66 \%$ and specificity was $50 \%$; for the right side, for the optimal cut-off value of 1.02 , sensitivity was $66 \%$ and specificity was $52 \%$.

Receiver operating characteristics yielded that gray-scale histogram analysis showed diagnostic

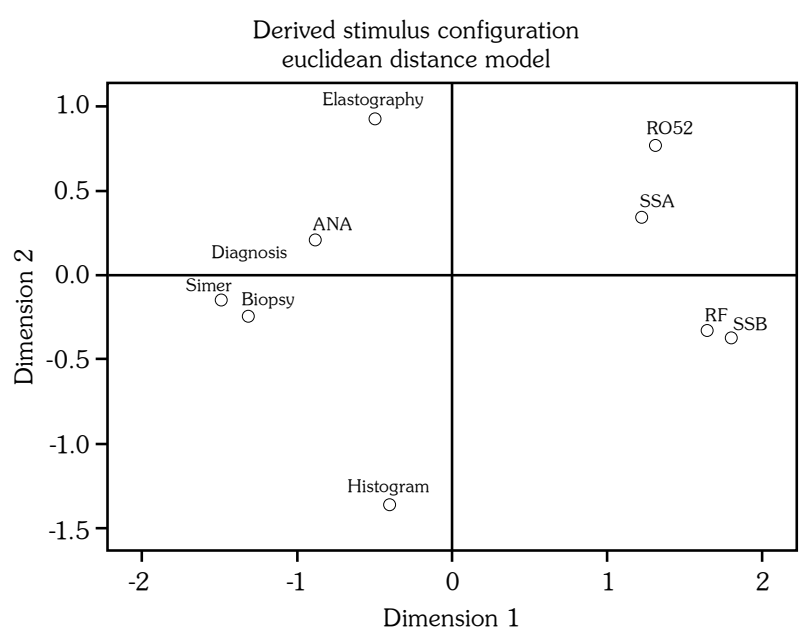

Figure 3. Multidimensional analysis of diagnostic parameters for diagnosis of Sjögren's syndromee.

ANA: Antinuclear antibodies; SSA: Sjögren's syndrome type A; RF: Rheumatoid factor; SSB: Sjögren's syndrome type B. 


\begin{tabular}{|c|c|c|c|}
\hline & Patient group & Control group & \\
\hline & Mean \pm SD & Mean \pm SD & $p$ \\
\hline BMUS for the right side & $1.6 \pm 1.4$ & $1.1 \pm 1.3$ & $<0.0001$ \\
\hline BMUS for the left side & $1.6 \pm 1.4$ & $1.1 \pm 1.3$ & $<0.0001$ \\
\hline Right parotid elastography & $1.3 \pm 0.8$ & $1.0 \pm 0.2$ & 0.009 \\
\hline Left parotid elastography & $1.4 \pm 0.9$ & $1.1 \pm 0.3$ & 0.020 \\
\hline Right parotid histogram & $1.3 \pm 0.5$ & $1.8 \pm 0.6$ & 0.001 \\
\hline Left parotid histogram & $1.4 \pm 0.8$ & $1.9 \pm 0.7$ & 0.002 \\
\hline
\end{tabular}

changes in 39/57 patients (68\%) and the SR values were diagnostic in 37/57 patients (64\%). In $13 / 57$ patients (22\%), elastography showed diagnostic changes and no abnormal changes were seen on gray-scale histogram analysis whereas in $12 / 57$ patients (21\%) with abnormal gray-scale histogram values, no diagnostic changes were determined on elastography.

The mean BMUS was $1.6 \pm 1.5$ and $1.6 \pm 1.5$ for the right and left sides, respectively, in the patient group and $1.1 \pm 1.3$ and $1.1 \pm 1.3$ for the right and left sides, respectively, in the control group. The BMUS of the patient group was significantly higher than that of the control group $(p<0.0001)$. Cronbach's alpha value of 0.976 for the composite intra- and inter-rater reliability/ consistency demonstrated excellent agreement between the two examiners and between different evaluations of the same examiners in the subjective BMUS assessments. Based on the consensus readings, the majority of the study participants were rated as Grade 0 and Grade 1 in the subjective evaluation of the echostructure of the parenchyma.

In the subjective ultrasonographic echogenicity assessment, of the total 105 individuals, 24 (23\%) (22 patients and 2 controls) on the right side and 25 (24\%) (23 patients and 2 controls) on the left side were rated as hypoechoic compared to the thyroid gland.

\section{DISCUSSION}

In this study, feasibility of sonoelastography and gray-scale histogram analysis as auxiliary ultrasonographic techniques were evaluated in the detection of parotid gland sonographic abnormalities in patients with SS. The mean histogram values obtained from gray-scale histogram analysis and the SR values obtained from sonoelastography were significantly different in normal parotid glands and those with SS. Moreover, the diagnostic performance of grayscale histogram analysis was higher than that of SR from sonoelastography in this study population.

The measurement of the distribution of the gray-levels within a particular area is a method of quantifying echogenicity of tissues. ${ }^{12}$ In daily clinical practice, gray-scale US images are evaluated by visual inspection, which has many disadvantages in terms of objectivity including operator dependency, US equipment, and setting dependency, which may influence the reproducibility of the assessments. Therefore, a relatively stable tissue is usually selected for comparison to assess the echogenicity of the organ of interest. However, since the reference organ may also not be normal, there is a need for more objective methods to quantify the echogenicity of the organ systems, which has a great impact on the diagnosis and follow-up of many diseases. There have been several studies on histogram analysis of gray-scale US images to detect a variety of diseases on different organ systems. While some have exported electronically-stored DICOM (Digital Imaging and Communications in Medicine) images and used external software to calculate the histogram values, there are several commercially available US systems with the histogram function to calculate gray-scale distribution width of $\mathrm{B}$-mode 
images. ${ }^{12,14,15}$ In the current study, an US system with integral histogram software was used, thus there was no need to export or transfer images to other software to calculate the histogram values, and this was a time-saving advantage.

Previous studies have shown that gray-scale histogram analysis of US images can distinguish echogenicity alterations of organ systems as a result of several diseases and those studies have reported this method to be a reliable and highly reproducible technique. ${ }^{12,14}$ To date, only a few studies have focused on quantification of the degree of hypoechogenicity of the parotid gland parenchyma. ${ }^{15,16}$ None of those studies found this technique to be useful in the differentiation of abnormal from normal glands. ${ }^{15,16}$ Unlike past studies, the results of the current study showed that the mean histogram values of abnormal parotid glands were significantly lower than those of normal glands reflecting the relative hypoechogeneity of parotid glands due to fibrosis developing secondary to Sjögren's disease compared to healthy glands. Further analyses of the data revealed satisfactory results for the diagnostic performance of this technique in the differentiation of abnormal glands from normal glands. The difference in the results of this study from previous studies may be explained by the study population differences in that the previous two studies recruited only diseased individuals and grouped patients on the basis of clinical findings and other auxiliary imaging tools were used including sialography and the analysis of US images was visual, which is a subjective evaluation. In the current study, a control group was formed of completely healthy individuals to compare with the patients. The lack of a healthy control group might have had a negative effect on the efficacy of the previous studies. Furthermore, due to the continuous improvements in scanner technology, the most recent images have higher resolution and those technical differences might also have played a role in the conflicting results of the current and previous studies. The superiority of gray-scale histogram analysis over the commonly used subjective visual method for US echogenicity assessment of the diseased glands can also be understood by looking at the positivity rates of both techniques in the patient group, which were $68 \%$ for the gray-scale histogram analysis and $38.5 \%(22 / 57)$ to $40 \%(23 / 57)$ for the subjective conventional method on the right and left sides, respectively. On the basis of this discrepancy, the use of objective gray-scale histogram analysis can be recommended as an alternative to conventional subjective visual assessment of the echogenicity of the parotid glands in patients with SS.

Elastography is an US technique that allows assessment of the stiffness of the organ, which may result from many disease states. Therefore, the organ affected either by neoplastic cells or by fibrosis can be evaluated on the basis of elastic properties. ${ }^{13}$ Even though sonoelastography has been proven to be useful in particular diseases such as liver fibrosis and breast cancer, there are controversial results in the literature on the benefits of sonoelastography for the diagnosis of salivary gland abnormalities in patients with SS. ${ }^{5,13,17}$ Dejaco et al. ${ }^{17}$ conducted a study on 45 patients with primary SS, 24 patients with sicca symptoms, and 11 healthy control subjects using real-time sonoelastography and reported that realtime sonoelastography might be useful, particularly in selected cases in which conventional US results were inconclusive as well as for the assessment of functional impairment of salivary glands. ${ }^{17}$ Similarly, in the current study, sonoelastography features supported the diagnosis of SS in 13 patients (22\%) although conventional US findings were non-diagnostic.

Furthermore, Hofauer et al. ${ }^{5}$ evaluated 100 patients grouped as patients with SS $(n=50)$ and a healthy control group $(n=50)$ according to AECG criteria using two different elastography techniques. Authors stated that neither real-time sonoelastography nor Virtual Touch ${ }^{\mathrm{TM}}$ imaging added more information to the conventional $\mathrm{B}$-mode US imaging. However, Virtual Touch quantification was reported to show promising results. ${ }^{5}$ In contrast, Zhang et al. ${ }^{18}$ studied 21 patients with SS and 11 healthy control subjects and stated that both Virtual Touch quantification and Virtual Touch tissue imaging were promising tools in the evaluation of major salivary glands for the clinical diagnosis of primary SS. ${ }^{18}$ Similar to the findings of Dejaco et al. ${ }^{17}$ and Zhang et al., ${ }^{18}$ the current study results demonstrated that SR values were significantly different in abnormal glands. The same sonoelastography was used as in the study of Dejaco et al., ${ }^{17}$ however, SR was analyzed, which is a semi-quantitative analysis to measure the elasticity of tissues, unlike the visual 
elasticity score evaluation. Regardless of the evaluation methods, both studies revealed that real-time sonoelastography can be an adjunctive tool for the evaluation of salivary glands in patients with SS. Furthermore, the SR values calculated in the current study from elastograms by drawing ROIs provided semiquantitative values which may be useful in the evaluation of the severity of the disease and follow-up of those patients together with the progression of the disease in the future. To the best of our knowledge, this is the first study to have calculated SR values for the parotid gland using real-time sonoelastography. Nevertheless, there is a need for further long-term studies to obtain more data on the efficacy of sonoleastography in the follow-up and treatment of these patients.

In the current study results, the only parameter that showed a significant correlation with the ultrasonographic evaluation was ANA autoantibodies. A different result of this study was that multidimensional analysis demonstrated that elastography and B-mode gray-scale histogram analysis values contributed more to the diagnosis of SS than those of ANA subgroup autoantibodies and RF. Previously, anti-RO/SS-A and anti-La/SS-B positivity has been reported as $40-75 \%$ and $23-52 \%$, respectively, while in the current study, these rates were $43 \%$ and $28 \%$, respectively. ${ }^{19}$ In the current study, the positivity rate of diagnostic changes was $68 \%$ for B mode gray-scale histogram analysis and $64 \%$ for sonoelastography. These results demonstrate that both gray-scale histogram analysis and sonoelastography methods were more beneficial than autoantibodies such as SS-A and SS-B, which have long been commonly used parameters in the diagnosis of SS. A previous study confirmed that US evaluation of salivary glands in SS strengthens the 2012 classification criteria. ${ }^{20}$ Recently, US imaging has started to be a part of the classification criteria for various rheumatic diseases and it may also be a part of the classification criteria for SS in the near future.

The Chisholm classification is based on the number of lymphocytic foci as a result of inflammation. ${ }^{10}$ This classification does not provide information about the parenchymal or extraparenchymal structure of the salivary glands. Both gray-scale histogram analysis, which measures the echogenicity of the glands, and sonoelastography, which assesses the stiffness of the glands, reveal the sclerotic changes thereby providing structural information about the salivary glands as a result of disease-related changes. This main difference between the methods may explain the lack of significant correlation of these two imaging techniques with the histopathological scores in early-stage patients. The reason that no significant correlation was determined between the disease activity indicators (ESSDAI and ESSPRI) and US imaging methods in the current study might be due to the low number of patients with systemic involvement as the disease was still in the early stage. Some of the previous studies have also reported a significant correlation between the dryness values (such as the Schirmer test.) and histopathological findings. ${ }^{21}$ In contrast, no correlation between those variables was determined in the current study. This could be attributed to study population differences as the current study patients were at the early stage of the disease. In some other past studies, patients with dryness symptoms have formed the control group. ${ }^{5,6,8}$ However, the salivary glands of those individuals might have been affected by non-inflammatory conditions which have the potential to alter the sonographic evaluation of the glands. None of the individuals in the current study had dryness complaints which can be considered to be a better representative of a healthy individual control group.

This study had some limitations. Since the control group comprised completely of healthy individuals, no salivary gland biopsies were performed and the autoantibodies test was not applied for ethical and economic reasons. Another limitation of the study was that due to time restraints, there was no second operator for the elastography and gray-scale histogram measurements, therefore, there could be no evaluation of inter-observer variability.

In conclusion, sonoelastography and grayscale histogram analysis of the parotid glands are beneficial and can be used as auxiliary ultrasonographic techniques to detect parotid gland sonographic abnormalities in patients with SS. Universal methods provided by the sonographic evaluation techniques may contribute to early diagnosis of the disease in addition to having a future place in the classification criteria. 


\section{Declaration of conflicting interests}

The authors declared no conflicts of interest with respect to the authorship and/or publication of this article.

\section{Funding}

The authors received no financial support for the research and/or authorship of this article.

\section{REFERENCES}

1. Cornec D, Devauchelle-Pensec V, Tobón GJ, Pers JO, Jousse-Joulin S, Saraux A. B cells in Sjögren's syndrome: from pathophysiology to diagnosis and treatment. J Autoimmun 2012;39:161-7.

2. Patel R, Shahane A. The epidemiology of Sjögren's syndrome. Clin Epidemiol 2014;6:247-55.

3. Vitali C, Bombardieri S, Jonsson R, Moutsopoulos HM, Alexander EL, Carsons SE, et al. Classification criteria for Sjögren's syndrome: a revised version of the European criteria proposed by the AmericanEuropean Consensus Group. Ann Rheum Dis 2002;61:554-8.

4. Shiboski SC, Shiboski CH, Criswell L, Baer A, Challacombe $\mathrm{S}$, Lanfranchi $\mathrm{H}$, et al. American College of Rheumatology classification criteria for Sjögren's syndrome: a data-driven, expert consensus approach in the Sjögren's International Collaborative Clinical Alliance cohort. Arthritis Care Res (Hoboken) 2012;64:475-87.

5. Hofauer B, Mansour N, Heiser C, Gahleitner C, Thuermel K, Bas M, et al. Sonoelastographic Modalities in the Evaluation of Salivary Gland Characteristics in Sjögren's Syndrome. Ultrasound Med Biol 2016;42:2130-9.

6. Baldini C, Luciano N, Tarantini G, Pascale R, Sernissi F, Mosca M, et al. Salivary gland ultrasonography: a highly specific tool for the early diagnosis of primary Sjögren's syndrome. Arthritis Res Ther 2015;17:146.

7. Çekiç B, Toslak IE, Şahintürk Y, Cekin AH, Koksel YK, Koroglu M, et al. Differentiating Transudative From Exudative Ascites Using Quantitative B-Mode Gray-Scale Ultrasound Histogram. AJR Am J Roentgenol 2017;209:313-319.

8. Seror R, Ravaud P, Mariette X, Bootsma H, Theander E, Hansen A, et al. EULAR Sjogren's Syndrome Patient Reported Index (ESSPRI): development of a consensus patient index for primary Sjogren's syndrome. Ann Rheum Dis 2011;70:968-72.

9. Seror R, Bootsma H, Saraux A, Bowman SJ, Theander E, Brun JG, et al. Defining disease activity states and clinically meaningful improvement in primary Sjögren's syndrome with EULAR primary Sjögren's syndrome disease activity (ESSDAI) and patient-reported indexes (ESSPRI). Ann Rheum Dis 2016;75:382-9.
10. Chisholm DM, Mason DK. Labial salivary gland biopsy in Sjögren's disease. J Clin Pathol 1968;21:656-60.

11. Hocevar A, Ambrozic A, Rozman B, Kveder T, Tomsic M. Ultrasonographic changes of major salivary glands in primary Sjogren's syndrome. Diagnostic value of a novel scoring system. Rheumatology (Oxford) 2005;44:768-72.

12. Maeda K, Utsu M, Kihaile PE. Quantification of sonographic echogenicity with grey-level histogram width: a clinical tissue characterization. Ultrasound Med Biol 1998;24:225-34.

13. Yağcı B, Erdem Toslak I, Çekiç B, Öz M, Karakaş BR, Akdemir M, et al. Differentiation between idiopathic granulomatous mastitis and malignant breast lesions using strain ratio on ultrasonic elastography. Diagn Interv Imaging 2017;98:685-91.

14. Kim GR, Kim EK, Kim SJ, Ha EJ, Yoo J, Lee HS, et al. Evaluation of underlying lymphocytic thyroiditis with histogram analysis using grayscale ultrasound images. J Ultrasound Med 2016;35:519-26.

15. Chikui T, Okamura K, Tokumori K, Nakamura S, Shimizu M, Koga M, et al. Quantitative analyses of sonographic images of the parotid gland in patients with Sjögren's syndrome. Ultrasound Med Biol 2006;32:617-22.

16. Chikui T, Shimizu M, Kawazu T, Okamura K, Shiraishi T, Yoshiura K. A quantitative analysis of sonographic images of the salivary gland: a comparison between sonographic and sialographic findings. Ultrasound Med Biol 2009;35:1257-64.

17. Dejaco C, De Zordo T, Heber D, Hartung W, Lipp R, Lutfi A, et al. Real-time sonoelastography of salivary glands for diagnosis and functional assessment of primary Sjögren's syndrome. Ultrasound Med Biol 2014;40:2759-67.

18. Zhang S, Zhu J, Zhang X, He J, Li J. Assessment of the Stiffness of Major Salivary Glands in Primary Sjögren's Syndrome through Quantitative Acoustic Radiation Force Impulse Imaging. Ultrasound Med Biol 2016;42:645-53.

19. Stefanski AL, Tomiak C, Pleyer U, Dietrich T, Burmester GR, Dörner T. The Diagnosis and Treatment of Sjögren's Syndrome. Dtsch Arztebl Int 2017;114:354-61.

20. Cornec D, Jousse-Joulin S, Marhadour T, Pers JO, Boisramé-Gastrin S, Renaudineau Y, et al. Salivary gland ultrasonography improves the diagnostic performance of the 2012 American College of Rheumatology classification criteria for Sjögren's syndrome. Rheumatology (Oxford) 2014;53:1604-7.

21. Jousse-Joulin S, Milic V, Jonsson MV, Plagou A, Theander E, Luciano $\mathrm{N}$, et al. Is salivary gland ultrasonography a useful tool in Sjögren's syndrome? A systematic review. Rheumatology (Oxford) 2016;55:789-800. 